\title{
ASTROCYTES IN COGNITIVE DYSFUNCTION
}

\author{
Mikhail V. Pletnikov
}

Johns Hopkins University School of Medicine, Baltimore, MD, 21287, USA mpetni1@jhu.edu

Astrocytes are the most abundant cells in the brain, and pathological changes in astrocytes likely contribute to cognitive impairment. I will present our recent studies of the role of astrocyte bioenergetics and glutamate metabolism in cognitive dysfunction. We have been using various experimental models to identify the molecular mechanisms underlying cognitive impairment because of abnormal neuronastrocyte metabolic coupling.

We have evaluated how a genetic risk factor, dominant-negative Cterminus-truncated human Disrupted-In-Schizophrenia-1 (DN-DISC1), selectively expressed in astrocytes would affect adult hippocampal neurogenesis and hippocampus-dependent learning and memory. We found that DN-DISC1 in astrocytes decreased neural progenitor proliferation and dendrite growth of newborn neurons and impaired hippocampusdependent learning and memory. As expression of DN-DISC1 leads to decreased secretion of D-serine, a co-agonist of NMDA receptor, we assessed possible ameliorative effects of chronic treatment with Dserine. We showed that $D$-serine rescued learning and memory and abnormal adult neurogenesis in DN-DISC1 mice, suggesting that genetic risk factors expressed in astrocytes could affect adult hippocampal neurogenesis and contribute to memory deficits.

We have also studied the role of DISC1 in metabolic functions of astrocytes and learning and memory. Both knockdown of Disc1 and expression of DN-DISC1 decreased mRNA and protein levels of glucose transporter 4 and glucose uptake by primary astrocytes. Reduced glucose uptake was associated with lower oxidative phosphorylation, glycolysis, and lactate production in the hippocampus in vivo. Lactate treatment rescued deficient learning and memory in DN-DISC1 male and female mice, suggesting that DISC1 influences lactate production in astrocytes to support neuronal activity required for cognitive function.

We have also tested the hypothesis that long-term cognitive impairment following adolescent cannabis use could be dependent on genetic risk factors expressed in astrocytes and could be mediated by action of delta-9-tetrahydrocannabinol ( $\triangle 9-T H C)$, a partial agonist of brain cannabinoid receptor 1 (CNR1) and a major cannabis constituent. We found that selective knockdown of Disc1 or expression of DN-DISC1 in astrocytes but not neurons and adolescent treatment with $\Delta 9-\mathrm{THC}$ synergistically impaired spatial learning and memory in adult mice. We determined that that DN-DISC1 and $\triangle 9$-THC synergistically activated the NF-kB-COX-2 pathway in astrocytes and decreased immunoreactivity of parvalbumin-positive pre-synaptic inhibitory boutons around pyramidal neurons of the CA3 area of the hippocampus. Blocking this inflammatory pathway with the COX-2 inhibitor, NS389, rescued cognitive abnormalities in DN-DISC1 mice. 
Overall, our studies provide new insights into contribution of astrocyte bioenergetics and metabolism to cognitive function and memory impairment associated with psychiatric disorders or drug abuse.

1. All animal studies were approved by the JHU ACUC and supported by DA-041208, MH-094268, $\mathrm{MH}-083728$ and the Brain and Behavior Research Foundation. 